\title{
Volumetric modulated arc therapy (VMAT) provides the conformality that enables three separate simultaneous pelvic malignancies to be treated radically-a case study
}

\begin{abstract}
Radiotherapy and surgery are both potential modalities for the definitive treatment of pelvic malignancies. Although surgery provides a histological specimen, enabling exact staging, radiotherapy is sometimes preferred as it provides patients the opportunity for organ conservation and therefore perhaps better quality post-treatment survivorship. Our case report details how three separate primary pelvic cancers (prostate, rectal, anal) in one patient were treated simultaneously with definitive radiotherapy. Patient was prescribed $80 \mathrm{~Gy}$ in 40 fractions to the planning target volume (PTV) prostate, 54 Gy to PTV anal canal, 45 Gy to the pelvis including PTV rectosigmoid junction and 36 Gy to the PTV inguinal lymph nodes that encompassed clinically negative nodes draining the anus, through the Volumetric Modulated Arc Therapy (VMAT) with 6MV photons. Mean doses to organs at risk (OAR) are $30.01 \mathrm{~Gy}$ to the bowel volume, $46.54 \mathrm{~Gy}$ to the bladder, $30.42 \mathrm{~Gy}$ to the femurs, and 61.84 Gy to the rectum. Radiation doses to the prostate and anal canal are consistent with conventional treatment doses with definitive radiotherapy. The rectal dose was accepted as part of the definitive treatment of rectal cancer following Endoscopic Mucosal Resection (EMR). This could only be achieved through the superior dose conformality of VMAT, maximising the dose given to tumour bearing PTV while minimising the dose to OARs which included normal pelvic structures. All three cancers remained under control at 4 years after treatment, with minimal late toxicity associated with the treatment received. Further RCTs in pelvic malignancies are needed to help clinicians and patients select the best treatments, to improve disease control while maintaining quality of survivorship.
\end{abstract}

Keywords: radiotherapy, volumetric modulated arc therapy, anal cancer, prostate cancer, rectal cancer, australia, case study
Volume 7 Issue 4 - 2020

\author{
Callista $\vee$ Raharjo,' Colin Chen, ${ }^{2}$ Francis \\ Lam, ${ }^{2}$ Siobhan O'Neill, ${ }^{2}$ John Almeida, ${ }^{2}$ Jenny \\ Turner ${ }^{3}$ Robyn Levingston, ${ }^{3}$ Anna O'Keefe, ${ }^{4}$ \\ lan Quin, ${ }^{4}$ Gerald B Fogarty ${ }^{5}$ \\ 'University of New South Wales Sydney, Australia \\ 2Prince of Wales Hospital,Australia \\ ${ }^{3}$ Department of Histopathology, Douglass Hanly Moir Pathology, \\ Australia \\ ${ }^{4}$ GenesisCare Radiation Oncology, St Vincents Hospital, Australia \\ ${ }^{5}$ GenesisCare Radiation Oncology, Mater Hospital, Australia
}

Correspondence: Gerald B Fogarty, GenesisCare Department of Radiation Oncology, Mater ospital Crows Nest, NSW, 2065,Australia, Email gerald.fogarty@genesiscare.com

Received: June 26, 2020 | Published: July 03, 2020
Abbreviations: RT, radiotherapy; 3D-CRT, three-dimensional conformal radiotherapy; IMRT, intensity modulated radiotherapy; VMAT, volumetric modulated arc therapy; EBRT, external beam radiotherapy; PSA, prostate-specific antigen; PSMA, prostatespecific membrane antigen; EMR, endoscopic mucosal resection; APR, abdominoperineal resection; SCC, squamous cell carcinoma; OAR, organ at risk; CEA, carcinoembryonic antigen; RTOG, radiation therapy oncology group; PTV, planning target volume; RCT, randomised controlled trial

\section{Introduction}

Surgery and radiotherapy (RT) can be competing modalities for the definitive treatment of some pelvic malignancies such as prostate cancer, ${ }^{1,2}$ early (stage IA, IB, IIA) cervical cancer, ${ }^{3,4}$ and early stage bladder cancer. ${ }^{5,6}$ Surgery provides a complete histological diagnosis, which aids exact staging, but involves tissue sacrifice with impact on the quality of survivorship. This is compared with the ability of RT to provide organ conservation. Those who choose surgery may suffer from decision regret. ${ }^{7-9}$ RT modalities and techniques have evolved over time, enabling better dose conformality. This means more doses to tumours and less to surrounding radiation sensitive normal structures, so more chance of cure for less side effects. Evolution from Three-dimensional Conformal Radiotherapy (3D-CRT) to Intensity
Modulated Radiotherapy (IMRT) and now to Volumetric Modulated Arc Therapy (VMAT) has enabled continuous beam modulation which is delivered in a rotational fashion quickly. Previously delivering therapeutic radical radiation to co-located primaries would be impossible as there would be too much overlap in 3DCRT radiation entrance and exit beams. We present a case where three separate simultaneous pelvic malignancies in the one patient were treated radically. This could only be done with VMAT.

\section{Case study}

A fit immunocompetent 74-year old male of Chinese descent was referred for definitive External Beam Radiotherapy (EBRT) for a high-risk, high-grade prostate cancer with Gleason score $4+5$ $=9$ in all 12 cores tested (Figure 1), and pre-treatment Prostatespecific Antigen (PSA) level elevated at $112 \mathrm{mg} / \mathrm{ml}$. Prostate-specific Membrane Antigen (PSMA) scan showed uptake in the prostate with SUV max of 23 in early images, SUV max of 58 in delayed images but no other disease. Co-morbidities included controlled cardiomyopathy, hypercholesterolemia, and Type II diabetes. Staging investigations of pelvic MRI and CT found an incidental polypoid lesion in the lower rectum and suspicious enlargement of the anus. Endoscopic biopsy showed suspicious cells in the rectal lesion and Endoscopic Mucosal Resection (EMR) of a $30 \mathrm{~mm}$ polypoid lesion 
at the rectosigmoid junction confirmed adenocarcinoma with positive margins at the higher portion of the rectum (Figure 2). The anal biopsy showed invasive Squamous Cell Carcinoma (SCC) suspicious for lymphovascular invasion at the anal verge (Figure 3). Definitive surgery for any of these lesions was declined. Injections of androgen deprivation therapy and radiosensitising Capecitabine (Xeloda) were started.

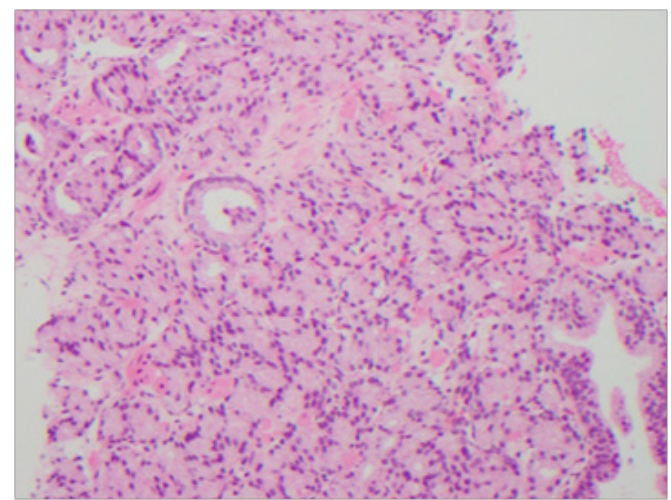

Figure I Histology of prostate cancer. Representative section consistent with adenocarcinoma of Grade Group 5 and Gleason Score of 9 (4+5).

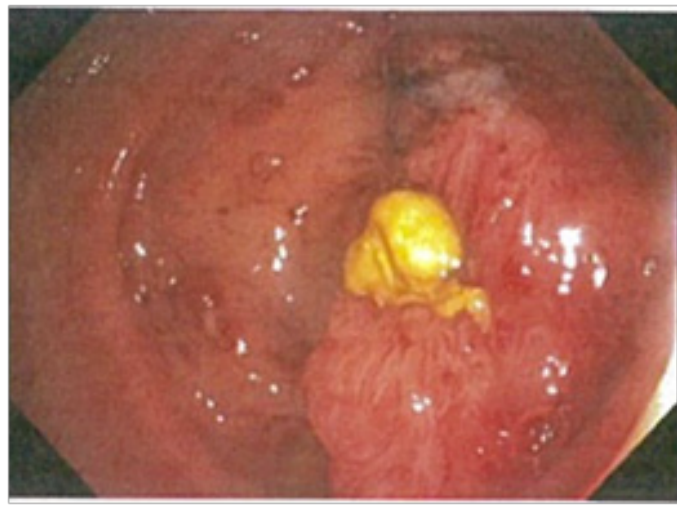

A

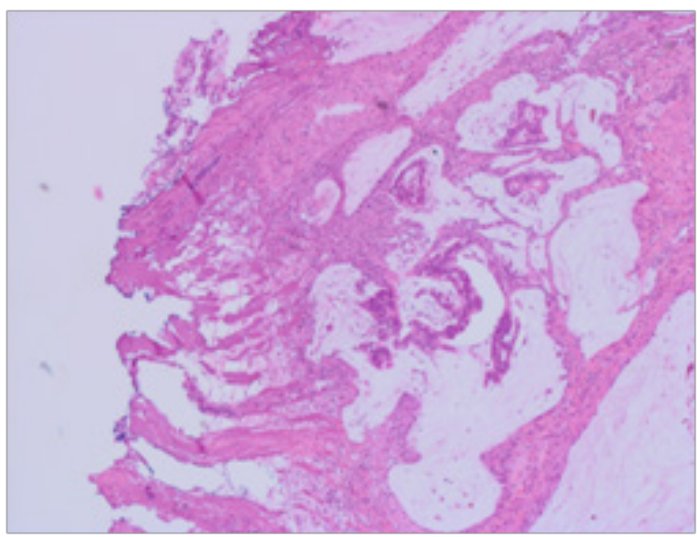

B

Figure 2 Macroscopic appearance and histology of rectal cancer. (A) Macroscopic appearance of $30 \mathrm{~mm}$ rectal lesion $12 \mathrm{~cm}$ from anal verge excised with Endoscopic Mucosal Resection. (B) Histology of rectal adenocarcinoma.
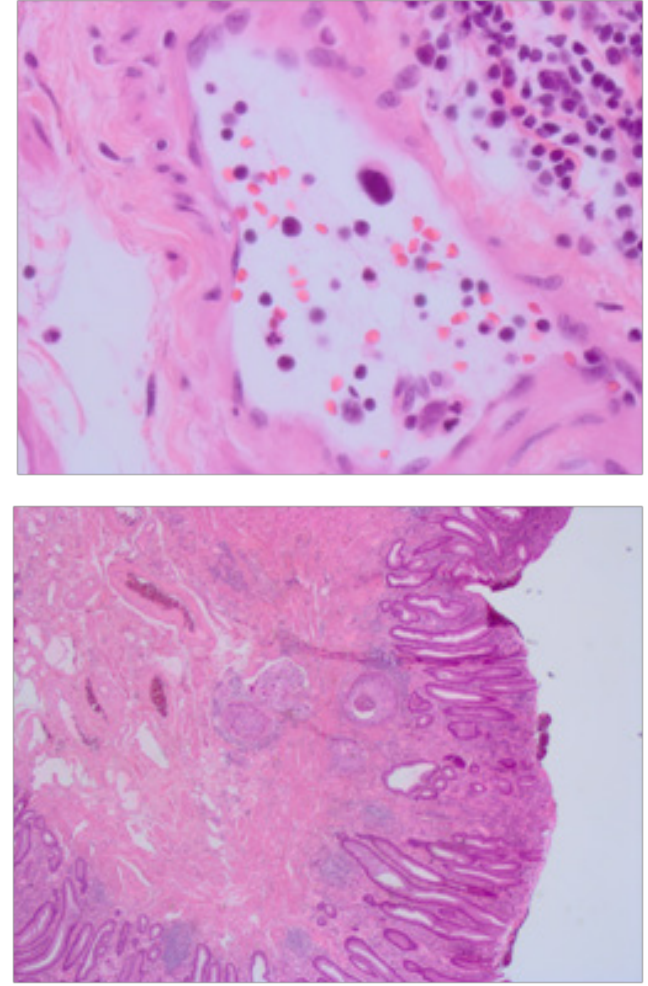

Figure 3 Histology of anal cancer. (A) Small focus of superficially invasive anal squamous cell carcinoma arising within a large area of high-grade squamous intraepithelial lesion. (B) Area suspicious for lymphovascular invasion.

RT planning, with target volume encompassing the anal region, prostate, rectosigmoid junction and pelvic and inguinal lymphatics, was performed. Patient was prescribed $80 \mathrm{~Gy}$ in 40 fractions to the planning target volume (PTV) prostate, 54 Gy to the PTV anal canal, 45 Gy to the PTV pelvis including rectosigmoid junction and 36 Gy to the PTV inguinal lymph nodes that included clinically negative draining anal nodes, through VMAT with $6 \mathrm{MV}$ photons. Mean dose to organs at risk (OARs) are $30.01 \mathrm{~Gy}$ to the bowel, $46.54 \mathrm{~Gy}$ to the bladder, $30.42 \mathrm{~Gy}$ to the femurs, and $61.84 \mathrm{~Gy}$ to the rectum. Radiation doses to the prostate and anal canal are consistent with conventional treatment doses with definitive radiotherapy. As patient refused Abdominoperineal Resection (APR) as initial definitive surgical treatment, the rectal radiotherapy dose was accepted as part of the definitive treatment of rectal cancer following EMR. Patient was agreeable to having salvage APR in the event that radical treatment of rectal cancer fails to achieve control of disease. Table 1 summarises the dose to each Planning Target Volume (PTV) and OAR. Figure 3 shows the cross-sectional dosimetry on the axial and midline sagittal scan. Figure 4 summarises the doses to tumour and OAR volumes through a Dose-Volume histogram. Radiotherapy to the prescription dose was completed on time. There were no acute toxicities that delayed treatment. To date, 4 years following RT completion, the patient has no late toxicities. His Prostate-specific Antigen (0.26 ng/ $\mathrm{ml})$ and Carcinoembryonic Antigen $(3.0 \mathrm{ng} / \mathrm{ml})$ levels remain within normal limits. 

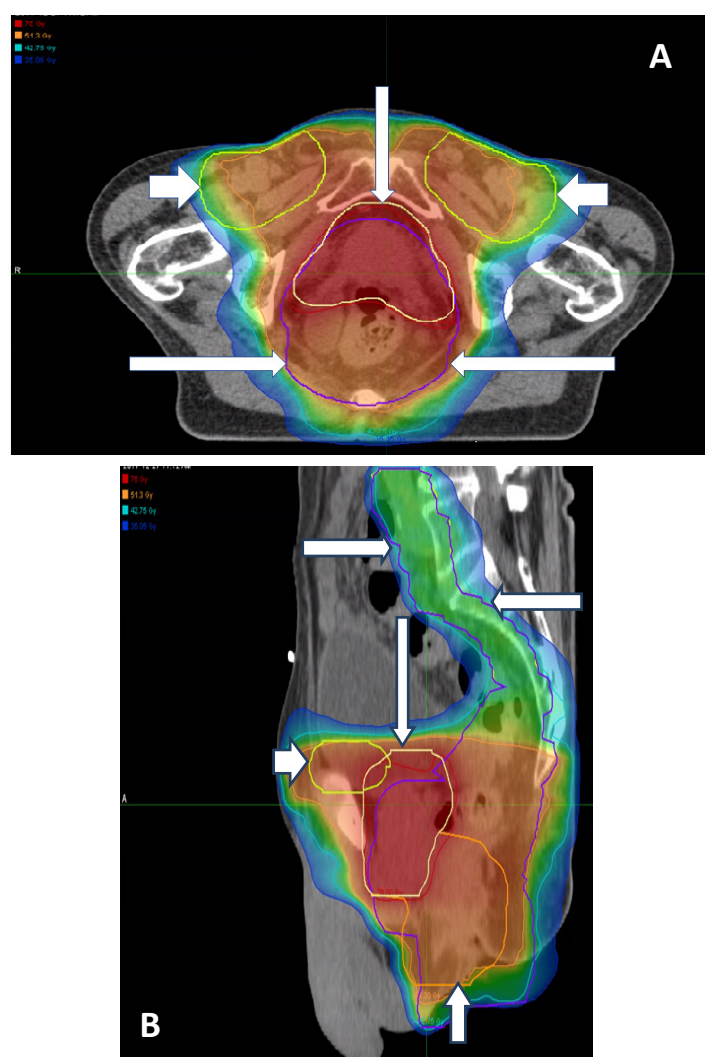

Figure 4 Cross-sectional dosimetry on axial (A) and midline sagittal (B) Scan. Short horizontal arrows- PTVI (Regional lymph nodes) - 36Gy; Long horizontal arrows - PTV2 (Pelvis) - 45Gy; Short vertical arrows - PTV3 (Anal Canal)- 54Gy; Long vertical arrows - PTV4 (Prostate)- 80Gy.

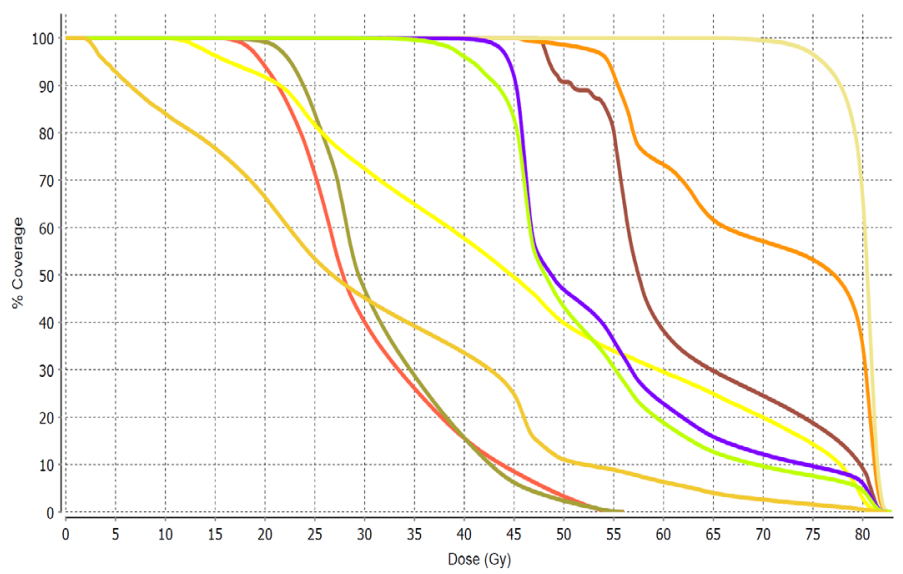

Figure 5 Dose Volume histogram with summary of doses to tumour and organ at risk (OAR) volumes. Short horizontal arrows - PTVI - 36Gy; Long horizontal arrows - PTV2 - 45Gy; Short vertical arrows - PTV3 - 54Gy; Long vertical arrows - PTV4 - 80Gy; Green Triangle - Bowels; Black Triangle Femurs; Red Triangle - Bladder; Brown Triangle - Rectum.

\section{Discussion}

We present a case where three separate simultaneous pelvic malignancies in the one patient were treated radically with VMAT.
RT has been proven to be a definitive treatment modality for prostate cancer, ${ }^{10}$ while concurrent chemoradiation is the mainstay treatment for anal SCCs following the Nigro Trial. ${ }^{11}$ However, the role of RT in rectal cancer treatment is less clearly defined. Endoscopic resection alone is not considered adequate treatment, with the standard surgical treatment being transabdominal surgery such as low anterior resection or abdominoperineal resection. ${ }^{12}$ For prostate cancer, the Australian and New Zealand Faculty of Radiation Oncology Genito-urinary Group recommended dose escalation to $78-80 \mathrm{~Gy}$, as it has been shown to improve tumour control, resulting in improved biochemical failure free survival when compared with conventional radiotherapy doses (68-70.2 Gy) in all risk groups. ${ }^{13}$ A minimum dose of 45 Gy is the currently recommended for radical treatment of anal SCC. ${ }^{14}$ Due to heterogeneity of results seen with higher boost doses of radiation up to $70 \mathrm{~Gy}$, conventional doses between 50.4 to $59.4 \mathrm{~Gy}$ are acceptable reserving doses at the higher end of the spectrum for bulkier anal disease. ${ }^{15-17}$ Nonetheless, higher RT doses to primary tumours inadvertently resulted in greater indirect exposure of radiotherapy doses to $\mathrm{OAR},{ }^{18,19}$ causing acute and late genitourinary and gastrointestinal toxicities.

VMAT enabled our patient to receive the recommended dose to each PTV as per current guidelines, while keeping the dose to OARs within the limits of dose constraints as outlined in Table 1. The patient is alive and well with no evidence of disease 4 years later. Late rectal and bladder toxicity was minimal at Grade $0-1$, as per Radiation Therapy Oncology Group (RTOG) Toxicity Criteria. ${ }^{20}$ Only the superior conformality of VMAT could deliver this. What is needed for this result is adequate dose for loco-regional control for all three cancers, but the dose to OAR is below that associated with late toxicity. It could be argued that the patient was radiation sensitive and that is why all three cancers were cured. However, if that were the case, OAR tissue would also be radiosensitive and late RT effects would be expected. It also should be acknowledged that good endoscopic resection of the rectal lesion in this patient enabled RT to definitively treat the microscopic residual disease. This highlights the potential for pre-radiotherapy surgical debulking as a treatment option for early rectal cancer with curative intent.

This patient declined definitive surgery for any lesions. Patients are driving what treatment they will get based on quality of life. For example, patients may choose to have radiotherapy to allow less intense surgical treatment which may facilitate sphincter-conservation ${ }^{21}$ and bladder-sparing surgeries ${ }^{22}$ when treating rectal cancer and bladder cancer respectively. RT preserves normal tissue leading to perhaps higher quality survivorship for the same oncological outcomes as surgery.

\section{Conclusion}

Perhaps combined modality treatments will be the best, with surgery for debulking allowing a lower RT dose to be used. This combined modality treatment has been successfully implemented in the treatment of breast cancer with radiotherapy after a breastconserving surgery. ${ }^{23,24}$ Randomised Controlled Trials (RCTs) are also currently being undertaken to establish the use of transoral surgery and lower dose post-operative radiotherapy to minimise treatment toxicity in Human Papilloma Virus-associated oropharyngeal squamous cell carcinoma. ${ }^{25-27}$ RCTs in pelvic malignancies are needed to help clinicians and patients to select the best treatments, which are tailored to the patients' individual cases. 
Table I Dose-volume distributions and constraints for target volume and organs at risk in prostate, rectal, anal cancer radiotherapy. *Radiation dose constraints to OAR are in accordance to RTOG recommendations

\begin{tabular}{|c|c|c|}
\hline Target volume & Dose distribution & Prescribed dose \\
\hline PTVI - Inguinal Lymph Nodes (2804.I5ml) & $\begin{array}{l}\text { Max Dose: } 83.03 \mathrm{~Gy} \\
\text { Mean Dose: } 52.48 \mathrm{~Gy}\end{array}$ & $36 \mathrm{~Gy}$ \\
\hline PTV2 - Pelvis including Rectosigmoid Junction(2040.09ml) & $\begin{array}{l}\text { Max Dose: } 83.03 \mathrm{~Gy} \\
\text { Mean Dose: } 54.13 \mathrm{~Gy}\end{array}$ & $45 \mathrm{~Gy}$ \\
\hline PTV3 - Anal Canal (36I.42ml) & $\begin{array}{l}\text { Max Dose: } 83.03 G y \\
\text { Mean Dose: } 70.63 G y\end{array}$ & $54 \mathrm{~Gy}$ \\
\hline PTV4 - Prostate (I89.49ml) & $\begin{array}{l}\text { Max Dose: } 83.03 \mathrm{~Gy} \\
\text { Mean Dose: } 79.93 \mathrm{~Gy}\end{array}$ & 80 Gy \\
\hline Organ-At-Risk (OAR) & Dose Constraints* & \\
\hline \multirow{4}{*}{ Bladder $(234.64 \mathrm{ml})$} & V65 Gy $<50 \%$ & \\
\hline & V70 Gy $<35 \%$ & \\
\hline & V75 Gy $<25 \%$ & \\
\hline & V80 Gy $<15 \%$ & \\
\hline \multirow{4}{*}{ Rectum $(4 \mathrm{I} .36 \mathrm{ml})$} & V60 Gy $<35 \%$ & \\
\hline & V65 Gy $<25 \%$ & \\
\hline & V70 Gy $<20 \%$ & \\
\hline & V75 Gy $<15 \%$ & \\
\hline Bowel (I830.39ml) & V45 Gy $<30 \%$ & \\
\hline Femur (I38.76ml) & V50 Gy $<5 \%$ & \\
\hline
\end{tabular}

\section{Acknowledgments}

We would like to thank the staff at Genesis Care and Prince of Wales Hospital for their contribution and commitment to the care of this patient, without which the writing of this case report would not be possible.

\section{Funding}

None.

\section{Conflicts/disclosures}

Authors declare that there is no conflict of interest.

\section{References}

1. Barocas DA, Alvarez J, Resnick MJ, et al. Association between radiation therapy, surgery, or observation for localized prostate cancer and patientreported outcomes after 3 years. JAMA. 2017;317(11):1126-1140.

2. Barocas DA, Penson DF. Functional Recovery Following Primary Treatment for Prostate Cancer: Update from the CEASAR Study. Eur Urol Focus. 2020;6(2):205-207.

3. Waggoner SE. Cervical cancer. Lancet. 2003;361(9376):2217-2225.

4. Hsu WC, Chung NN, Chen YC, et al. Comparison of surgery or radiotherapy on complications and quality of life in patients with the stage IB and IIA uterine cervical cancer. Gynecol Oncol. 2009;115(1):41-45.

5. Elzbieta van der Steen-Banasik, Martine Ploeg, Johannes A Witjes, et al. Brachytherapy versus cystectomy in solitary bladder cancer: a case control, multicentre, East-Netherlands study. Radiother Oncol. 2009;93(2):352-357.

6. Rodel C, Grabenbauer GG, Kuhn R, et al. Combined-modality treatment and selective organ preservation in invasive bladder cancer: long-term results. J Clin Oncol. 2002;20(14):3061-3071.

7. Shakespeare TP, Chin S, Manuel L, et al. Long-term decision regret after post-prostatectomy image-guided intensity-modulated radiotherapy. $J$ Med Imaging Radiat Oncol. 2017;61(1):141-145.

8. Check DK, Leo MC, Banegas MP, et al. Decision Regret Related to Urinary Diversion Choice among Patients Treated with Cystectomy. $J$ Urol. 2020;203(1):159-163.

9. Shariat SF, Margulis V, Lotan Y, et al. Nomograms for bladder cancer. European urology. 2008;54(1):41-53.

10. Lee RJ, Sause WT. Surgically staged patients with prostatic carcinoma treated with definitive radiotherapy: fifteen-year results. Urology. 1994;43(5):640-644.

11. Benson A, Venook A, Al-Hawary M, et al. Anal Carcinoma, Version 2.2018, NCCN Clinical Practice Guidelines in Oncology. JNCCN. 2018;16:852-871.

12. Feeney G, Sehgal R, Sheehan M, et al. Neoadjuvant radiotherapy for rectal cancer management. World J Gastroenterol. 2019;25(33):4850.

13. Hayden AJ, Martin JM, Kneebone AB, et al. Australian \& New Zealand Faculty of Radiation Oncology Genito-Urinary Group: 2010 consensus guidelines for definitive external beam radiotherapy for prostate carcinoma. J Med Imaging Radiat Oncol. 2010;54(6):513-525. 
14. Scher ED, Ahmed I, Yue NJ, et al. Technical aspects of radiation therapy for anal cancer. J Gastrointest Oncol. 2014;5(3):198.

15. Ortholan C, Ramaioli A, Peiffert D, et al. Anal canal carcinoma: earlystage tumors $\leq 10 \mathrm{~mm}$ (T1 or Tis): therapeutic options and origina pattern of local failure after radiotherapy. Int J Radiat Oncol Biol Phys. 2005;62(2):479-485.

16. Huang K, Haas-Kogan D, Weinberg V, et al. Higher radiation dose with a shorter treatment duration improves outcome for locally advanced carcinoma of anal canal. World J Gastroenterol. 2007;13(6):895-900.

17. Peiffert D, Tournier-Rangeard L, Gérard JP, et al. Induction chemotherapy and dose intensification of the radiation boost in locally advanced anal canal carcinoma: final analysis of the randomized UNICANCER ACCORD 03 trial. J Clin Oncol. 2012;30(16):1941-1948.

18. Kuban DA, Tucker SL, Dong L, et al. Long-term results of the MD Anderson randomized dose-escalation trial for prostate cancer. Int $J$ Radiat Oncol Biol Phys. 2008;70(1):67-74.

19. Chan LW, Xia P, Gottschalk AR, et al. Proposed rectal dose constraints for patients undergoing definitive whole pelvic radiotherapy for clinically localized prostate cancer. Int J Radiat Oncol Biol Phys. 2008;72(1):69-77.

20. Cox JD, Stetz J, Pajak TF. Toxicity criteria of the radiation therapy oncology group (RTOG) and the European organization for research and treatment of cancer (EORTC). Int $J$ Radiat Oncol Biol Phys. 1995;31(5):1341-1346.

21. Rengan R, Paty P, Wong WD, et al. Distal cT2N0 rectal cancer: is there an alternative to abdominoperineal resection? Journal of Clinical Oncology. 2005;23(22):4905-4912.
22. Berry DL, Nayak M, Halpenny B, et al. Treatment Decision Making in Patients with Bladder Cancer. Bladder cancer. 2015;1(2):151-158.

23. Darby S, McGale P, Correa C, et al. Effect of radiotherapy after breastconserving surgery on 10-year recurrence and 15-year breast cancer death: meta-analysis of individual patient data for 10,801 women in 17 randomised trials. Lancet. 2011;378(9804):1707-1716.

24. Viani GA, Stefano EJ, Afonso SL, et al. Breast-conserving surgery with or without radiotherapy in women with ductal carcinoma in situ: a metaanalysis of randomized trials. Radiation oncology. 2007;2:28.

25. Nichols AC, Lang P, Prisman E, et al. Treatment de-escalation for HPVassociated oropharyngeal squamous cell carcinoma with radiotherapy vs. trans-oral surgery (ORATOR2): study protocol for a randomized phase II trial. BMC Cancer. 2020;20(1):125.

26. Owadally W, Hurt C, Timmins H, et al. PATHOS: a phase II/III trial of risk-stratified, reduced intensity adjuvant treatment in patients undergoing transoral surgery for Human papillomavirus (HPV) positive oropharyngeal cancer. BMC cancer. 2015; 15:602.

27. Mirghani H, Amen F, Blanchard P, et al. Treatment de-escalation in HPVpositive oropharyngeal carcinoma: ongoing trials, critical issues and perspectives. Int J Cancer. 2015;136(7):1494-503. 